\title{
Obesity Is an Independent Predictor of Poor Survival in Metastatic Breast Cancer: Retrospective Analysis of a Patient Cohort Whose Treatment Included High-Dose Chemotherapy and Autologous Stem Cell Support
}

\author{
A. von Drygalski, ${ }^{1,2}$ T. B. Tran, ${ }^{1}$ K. Messer, ${ }^{3}$ M. Pu, ${ }^{3}$ S. Corringham, ${ }^{4}$ \\ C. Nelson, ${ }^{4}$ and E. D. Ball ${ }^{4}$ \\ ${ }^{1}$ Division of Hematology/Oncology, Department of Medicine, UCSD, CA 92093, USA \\ ${ }^{2}$ Moores Cancer Center, 3855 Health Sciences Drive, La Jolla, Ca 92093-0829, USA \\ ${ }^{3}$ Division of Biostatistics and Bioinformatics, Department of Family and Preventive Medicine, UCSD, CA 92093, USA \\ ${ }^{4}$ Division of Bone and Marrow Transplantation, Department of Medicine, UCSD, CA 92093, USA
}

Correspondence should be addressed to A. von Drygalski, avondrygalski@ucsd.edu

Received 4 April 2011; Accepted 5 May 2011

Academic Editor: Filippo Montemurro

Copyright ( 12011 A. von Drygalski et al. This is an open access article distributed under the Creative Commons Attribution License, which permits unrestricted use, distribution, and reproduction in any medium, provided the original work is properly cited.

\begin{abstract}
The purpose of the study was to identify predictors of long-term survival in metastatic breast cancer (MBC). A cohort of 96 patients, who received high-dose chemotherapy with autologous stem cell support (HD-ASCT) as part of their treatment, was analyzed. Percent long-term survival at 10 years was 24.5\% (CI 17.2-34.9\%) when metastasis was diagnosed and $14.4 \%$ (CI $8.7-$ $23.9 \%$ ) when MBC was diagnosed. Survival was impacted significantly by body mass index (BMI). Median overall survival from initial diagnosis or from time of metastasis for patients with BMIs $\leq 30$ and $>30$ (obese) was 7.1 (CI 4.4-8.7) and 3.2 years (2.416.75 ), respectively, or 3.2 or 2.3 years (all $P=0.02$ ). Also, obesity was the only independent patient-related predictor of time to metastasis and of survival. While obesity is linked with poor outcomes in earlier stages of breast cancer, this has not been previously reported for $\mathrm{MBC}$.
\end{abstract}

\section{Introduction}

Breast cancer is the second leading cause of cancer deaths in women today after lung cancer. Metastatic breast cancer (MBC) is deemed incurable and median survival for patients with cancer that is estrogen receptor (ER) negative or no longer hormonally responsive is only 18 to 24 months $[1,2]$.

A variety of molecularly targeted drugs have been developed for MBC, but to date only, trastuzumab (Herceptin, Genentech, CA, USA) has shown an overall survival (OS) benefit of several months [3-5]. Clearly, more effective treatment strategies and/or improved patient selection are urgently needed. In the 1990s, high dose chemotherapy followed by autologous stem cell transplantation (HD-ASCT) was pioneered to improve survival. The initial feasibility studies in small cohorts of patients with advanced stage dis- ease showed improved survival when compared to historical controls [6-9]. Subsequent prospective randomized clinical trials comparing HD-ASCT to standard chemotherapy resulted in improved PFS, but failed to demonstrate OS benefit [10-14]. The results of the last trial of these series with 386 patients with metastatic breast cancer employing HDASCT were reported most recently and confirmed the results of earlier studies [15]. All together, the lack of convincing survival data at the expense of high toxicity resulted in the demise of HD-ASCT as a treatment modality of MBC.

However, while the vast majority of patients with MBC will succumb to their disease within a relatively short period of time, $5-10 \%$ of patients live longer than 5 years, and observational data suggest that $1-3 \%$ of patients treated with conventional chemotherapy and/or hormonal manipulation may experience long-term survival beyond $10-15$ years 
[16-21]. Quite surprisingly, the most impressive long-term survival rates in excess of $10 \%$ were reported with HD-ASCT [22-24]. Response to treatment, site of metastases, hormone receptor status, performance status, and short initial diseasefree interval were important prognostic factors for survival after HD-ASCT and corresponded to prognostic factors known from treatment with conventional, less aggressive therapies [25-27]. However, the observation periods in all those studies rarely exceeded 5 years.

We have studied long-term survival rates as well as the distinct disease- and patient-related characteristics that would be predictive of long-term survival in a cohort of patients with MBC, who were treated at our institution between 1989 and 1999. Treatments for all patients were not limited to, but included HD-ASCT.

\section{Methods}

2.1. Patient Population and Data Extracted. Records of all patients in the bone marrow transplant registry at UCSD treated with HD-ASCT for MBC between 1989 and 1999 were retrospectively reviewed. All patients were females. Patients were followed for a median of 65 months from diagnosis (range 10.4-255.0 months; quartiles 36.7-109.3 months). No patient was excluded from analysis. Data acquisition and patient confidentiality safeguards were approved by the Institutional Review Board. Age, race, stage at diagnosis, histology, estrogen receptor (ER) and menopausal status, body mass index (BMI) in $\mathrm{kg} / \mathrm{m}^{2}$ at the time of HD-ASCT, time to transplant and death, site of metastasis, and disease status prior to HD-ASCT were extracted. Since all transplants were carried out prior to routine assessment of Her-2/neu receptor status, this information was not part of our analysis. A BMI > 30 was defined as obesity (http://www.cdc.gov/). Menopause was defined clinically by absence of menstruation for more than 12 months (http://www.cancer.gov/).

2.2. Treatments. High dose chemotherapy prior to stem cell support was platinum based in combination with thiotepa/ etoposide, ifosfamide/etoposide, cyclophosphamide/BCNU, cyclophosphamide/mitoxantrone, or cyclophosphamide/thiotepa. Dosing was strictly weight-based. Body surface area was allowed to exceed $2 \mathrm{~m}^{2}$. Ten patients received HD-ASCT in first line; all other patients received at least one line of treatment prior to HD-ASCT (median 1; range 0-4). Hormonal treatments for ER+ patients were administered at physicians' discretion. Autologous stem cell rescue was performed with harvested bone marrow cells in 6 patients. The other 89 patients received peripherally mobilized stem cells, 23 of which received grafts supplemented with harvested bone marrow cells. Mobilization was achieved with granulocyte- or granulocyte/macrophage-colony stimulating factor with or without cyclophosphamide.

2.3. Assessment of Disease Status. Extent of metastatic disease was determined within 12 weeks prior to and within 612 weeks after HD-ASCT by imaging and physical exam. Thereafter, assessment of disease status was prompted by
TABLe 1: Patient and disease characteristics.

\begin{tabular}{|c|c|}
\hline & $N$ \\
\hline \multicolumn{2}{|l|}{ Race } \\
\hline White & 81 \\
\hline Hispanic & 7 \\
\hline Asian & 4 \\
\hline African-American & 4 \\
\hline \multicolumn{2}{|l|}{ Age at metastasis } \\
\hline$<$ age 40 & 19 \\
\hline$\geq$ age 40 & 77 \\
\hline \multicolumn{2}{|c|}{ Menopausal status at diagnosis } \\
\hline Premenopausal & 28 \\
\hline Postmenopausal & 68 \\
\hline Unknown & 2 \\
\hline \multicolumn{2}{|l|}{ BMI at metastasis } \\
\hline$<30$ & 73 \\
\hline$\geq 30$ & 23 \\
\hline \multicolumn{2}{|l|}{ Stage at diagnosis } \\
\hline Stage I & 21 \\
\hline Stage II & 43 \\
\hline Stage III & 23 \\
\hline Stage IV & 8 \\
\hline Unknown & 1 \\
\hline \multicolumn{2}{|l|}{ Histology } \\
\hline Infiltrating ductal & 79 \\
\hline Infiltrating lobular & 10 \\
\hline Unknown & 7 \\
\hline \multicolumn{2}{|l|}{ Estrogen receptor status } \\
\hline Positive & 57 \\
\hline Negative & 36 \\
\hline Unknown & 3 \\
\hline \multicolumn{2}{|l|}{ Site of metastasis } \\
\hline Visceral & 38 \\
\hline Bone & 28 \\
\hline Lymph nodes & 14 \\
\hline Local & 16 \\
\hline
\end{tabular}

patient symptoms and carried out at physicians' discretion. Patients were grouped according to site of metastasis: in visceral or bone metastasis, or local or nodal recurrence. If multiple sites of metastasis were present, assignment to one metastatic site was prioritized as follows: visceral $>$ bone $>$ nodal > local. Complete response $(\mathrm{CR})$, partial response $(\mathrm{PR})$, stable disease $(\mathrm{SD})$, and progressive disease $(\mathrm{PD})$ were determined according to RECIST criteria.

2.4. Statistical Analysis. Multiple linear regression models were used to explore the association between a continuous outcome and predictors of interest. Residual analysis was performed to check for outliers, normality assumption, and heteroscedasticity. Transformation was done on the outcomes if necessary. Brookmeyer and Crowley's 95\% confidence intervals were used for median survival times [28]. 


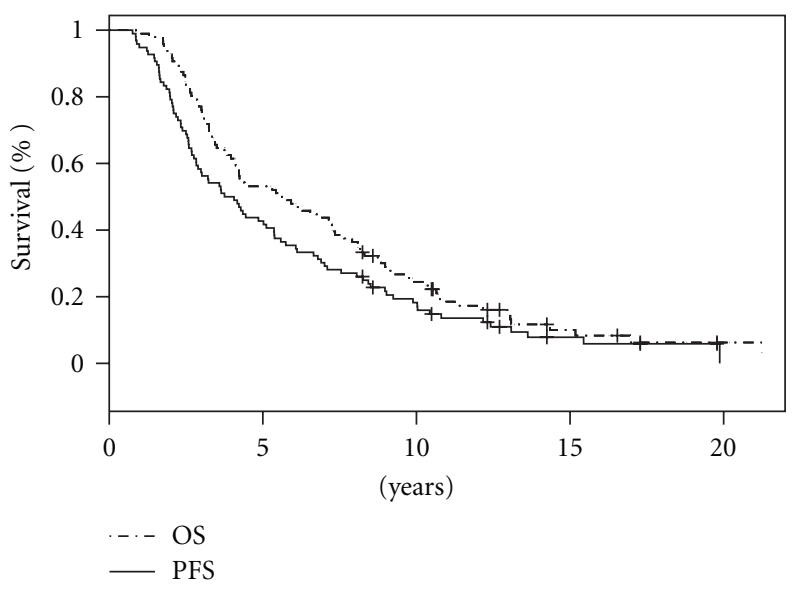

(a) Survival from initial diagnosis

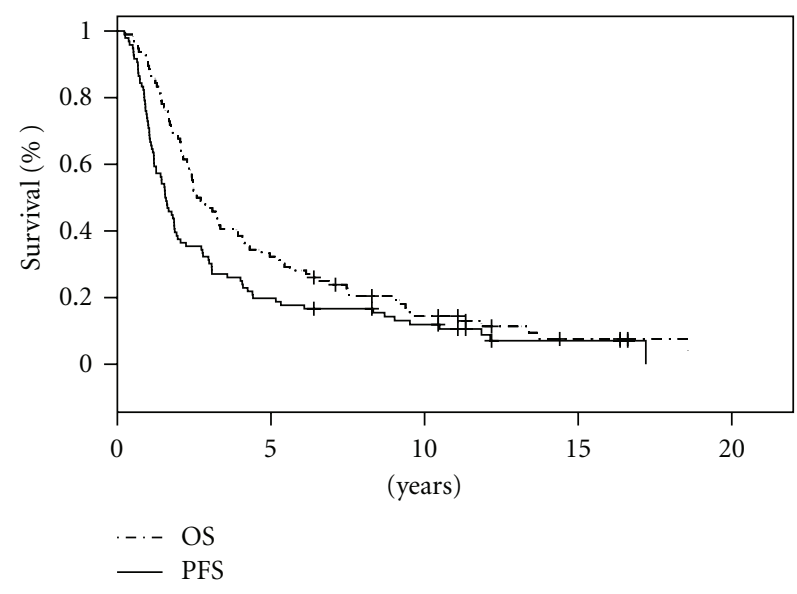

(b) Survival from diagnosis of metastasis

Figure 1: Overall survival and progression-free survival for all patients $(n=96)$ from initial diagnosis (a) and diagnosis of metastasis (b). Survival was estimated by Kaplan-Meier method.

PFS and OS were estimated using Kaplan-Meier method. Cox models were used to identify independent predictors of a time-to-event variable and Schoenfeld tests were used to test for proportional hazard assumptions. Predictors of interest with $P<0.2$ in the univariate analysis were included in an initial model and a manual backward selection was made based on the Wald test $P$ values. One subject with an unknown stage value was excluded from the final models. All analyses used the statistical package R version 2.5.1, 2007. (http://www.r-project.org/).

\section{Results}

3.1. Patient and Disease Characteristics. Patient-related characteristics are listed in Table 1. Median age at initial diagnosis was 43 years (quartiles 38-49) and 47 years (quartiles 41-43) at metastasis. Median time from initial diagnosis to documentation of metastasis was 29 months (quartiles 16.6-63.7), and from metastasis to HD-ASCT 6.6 months (quartiles 4.5-16.6), respectively. Twelve patients received HD-ASCT as their first line of treatment, whereas 84 patients had at least one prior line of chemotherapy for metastatic disease (48 patients with 1 line, 24 with 2 lines, 10 patients with 3 lines, and 2 patients with 4 lines of chemotherapy). Median BMI at metastasis was 25 (quartiles 21.4-29.6) and $24 \%$ of patients were obese (BMI > 30).

3.2. Survival Rates and Influence of BMI on Survival. For all patients $(n=96)$, median PFS and OS were 3.9 years (CI 2.95.4 ) and 5.6 years (CI 4.1-7.4) after initial diagnosis, and 1.6 years (CI 1.3-1.9) and 2.7 years (CI 2.3-3.9) after diagnosis of metastatic disease, respectively (Figures 1(a) and 1(b)). Survival rates at 10 years were $24.5 \%$ (CI 17.2-34.9\%) from diagnosis and 14.4\% (CI 8.7, 23.9\%) from when metastatic disease was diagnosed. As depicted in Figure 2, PFS and OS differed significantly for patients with a BMI $\leq 30$ and $>30$. At diagnosis, median PFS for patients with BMIs $\leq 30$ $(n=73)$ and $>30(n=23)$ was 4.4 (CI 3.6-6.7) and 2.5 years (CI 1.7-4.9), and median OS was 7.1 (CI 4.48.7 ) and 3.2 years (2.41-6.75), respectively (Figure 2(a) and $2(\mathrm{c})$; all $P$ values $=0.001$; univariate). Once metastasis was diagnosed, median PFS for patients with BMIs $\leq 30$ and $>30$ was 1.8 (CI 1.44-2.77) and 1.04 years (0.9-1.96) years, and median OS was 3.20 (CI 2.42-4.30) and 2.30 years (CI 1.564.96), respectively (Figure $2(\mathrm{~b})$ and $2(\mathrm{~d})$; all $P$ values $<0.02$; univariate).

3.3. Independent Predictors of Time from Initial Diagnosis to Metastasis. Ethnicity, stage at diagnosis, histology, site of metastasis, remission prior to HD-ASCT, menopausal status, ages at HD-ASCT and at diagnosis ( $<$ age 40 versus $\geq$ age 40 ), BMI ( $\leq 30$ versus $>30$ ), and ER status were evaluated as independent predictors of PFS and OS. In a univariate analysis, BMI, stage at initial diagnosis, and histology were predictive of time from initial diagnosis to metastatic disease (all $P$ values $<0.01$ ). As depicted in Table 2 , only the initial stage at diagnosis and BMI were confirmed as independent predictors in a multivariate multilinear regression analysis. Time to metastasis was significantly shorter for patients with a BMI > 30 (Table 2). Median time to metastasis was 1.14 years for patients with a BMI $>30$ compared to 1.85 years for patients with a $\mathrm{BMI} \leq 30(P<0.04$ for time to metastasis $)$. Times to metastasis were similar for stage I or II disease (approximately 2 years), but time to metastasis shortened significantly compared to stage I or II disease when stage III was present at diagnosis $(0.72$ years, $P<0.0006)$.

3.4. Independent Predictors of Survival from Metastasis. Stage at diagnosis, ER-status, site of metastasis, remission status prior to HD-ASCT, and BMI were considered for multivariate analysis since univariate $P$ values were $<0.02$. Only BMI, stage at diagnosis and site of metastasis were confirmed to be independent predictors of survival at the time of metastasis (Table 3). Patients with a BMI > 30 had a significantly higher 
TABLE 2: Independent risk factors for time from initial diagnosis to metastasis.

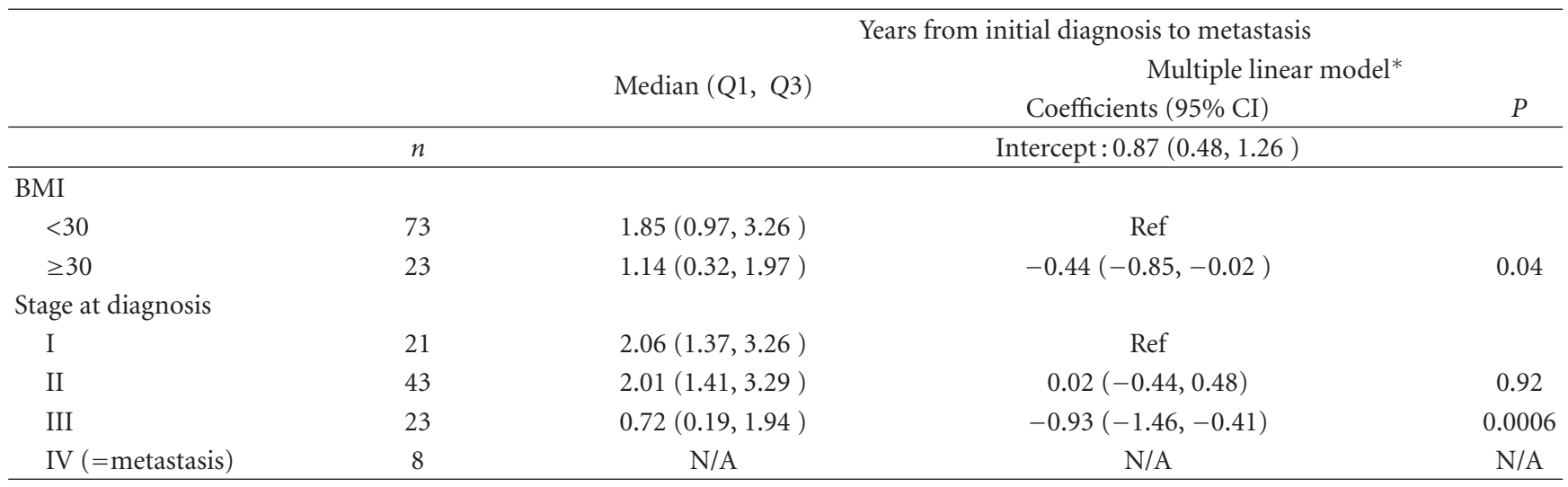

$* \log ($ years from initial diagnosis to metastasis +0.1$)$ was used as the outcome.

TABLE 3: Independent risk factors for progression-free survival (PFS) and overall survival (OS) at diagnosis of metastasis.

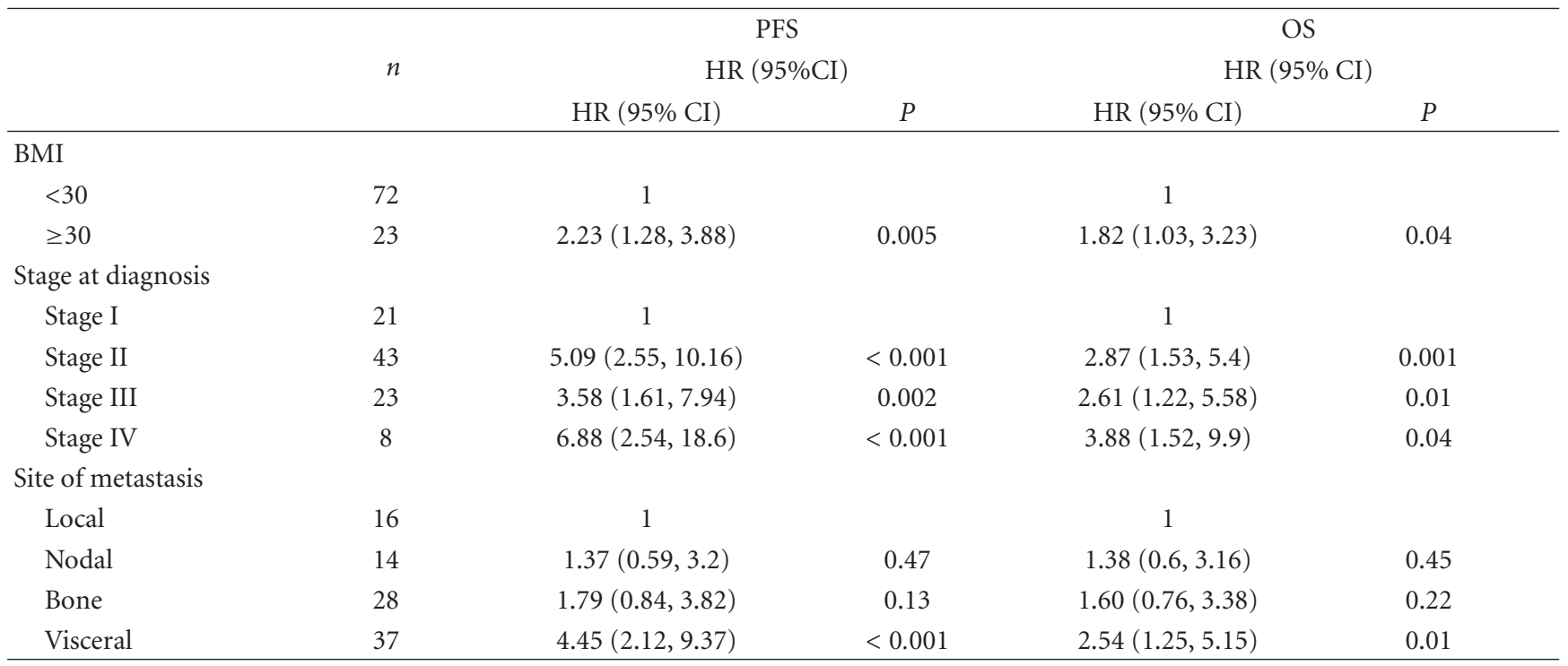

hazard of progression and death compared to patients with a $\mathrm{BMI} \leq 30$ ( $\mathrm{HR}$ for progression $=2.23 ; P=0.005$; $\mathrm{HR}$ for death $=1.82 ; P=0.04)$. Also, once metastasis was diagnosed, hazards of progression and death increased significantly with increasing stages present at diagnosis (compared to stage I all $P$ values $<0.05$; Table 3 ) or with visceral disease.

\section{Discussion}

In the 1990s, high HD-ASCT was thought to be a beneficial treatment option in $\mathrm{MBC}$, based on the hypothesis that significant dose escalation would improve the efficacy of chemotherapy. When several randomized trials demonstrated that OS was not significantly improved [1-15]. HDASCT was abandoned. Since cure could not be achieved with aggressive treatment, the school of thought changed and sequential single agent chemotherapy with or without biological targeted therapies and /or hormonal manipulation in $\mathrm{ER}+$ patients has become the preferred way of symptom control [2, 29].

However, a proportion of patients with MBC can achieve long-term survival which has been reported to exceed $10 \%$ with more aggressive chemotherapy such as HD-ASCT [2224]. We were specifically interested if such high percentages of survival could be maintained beyond 10 years, at which point it is possible that at least some of these patients may be cured of their disease. Second, we studied the patientand disease-specific characteristics predictive of survival. For both questions we analyzed our institutional cohort of 96 patients with $\mathrm{MBC}$ who had received HD-ASCT as part of their treatment algorithm. Since the last patient was transplanted in 1999, each patient had a minimum followup of 10 years.

First, we did find long-term survival in excess of 10 years. Survival rates at 10 years were $24.5 \%$ from initial diagnosis and $14.4 \%$ from when metastatic disease was diagnosed. 


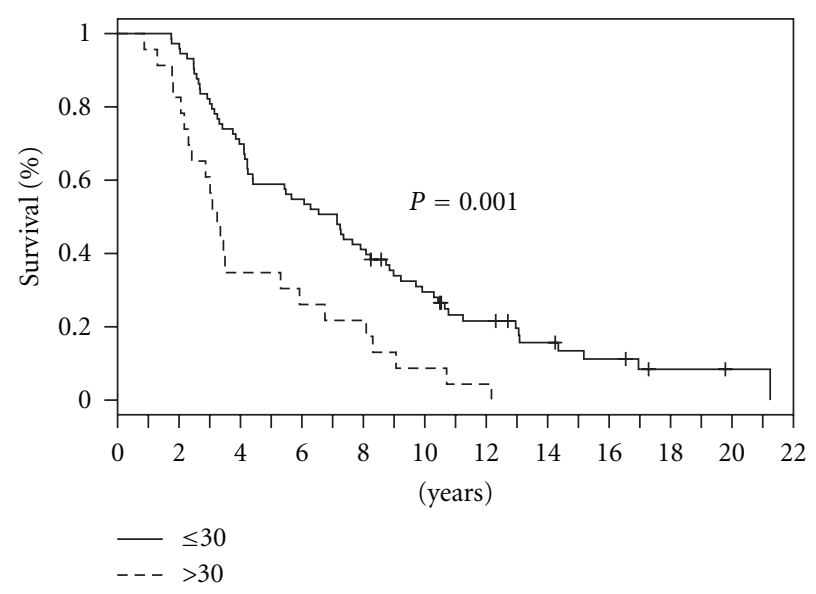

(a) OS from initial diagnosis

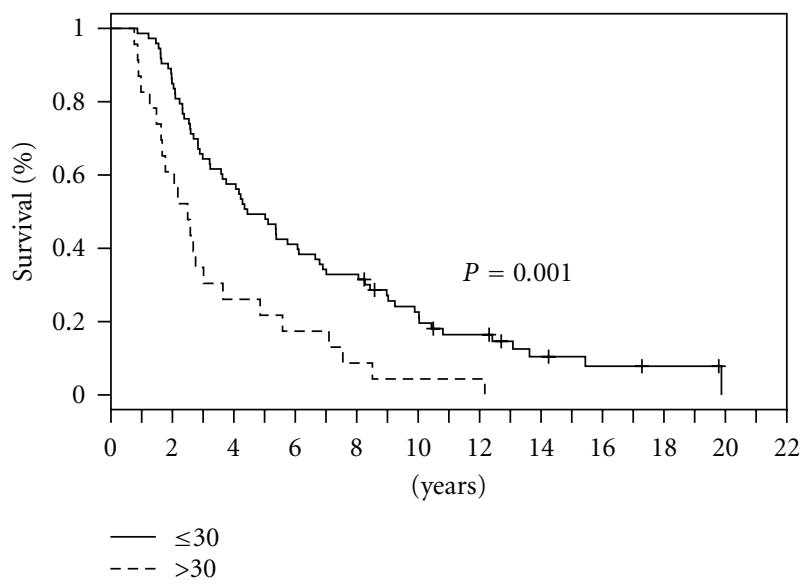

(c) PFS from initial diagnosis

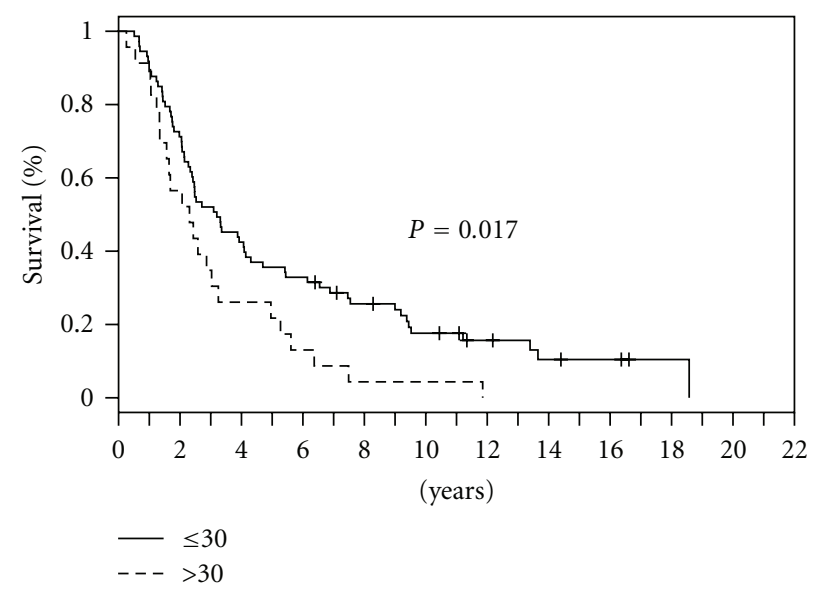

(b) OS from metastasis

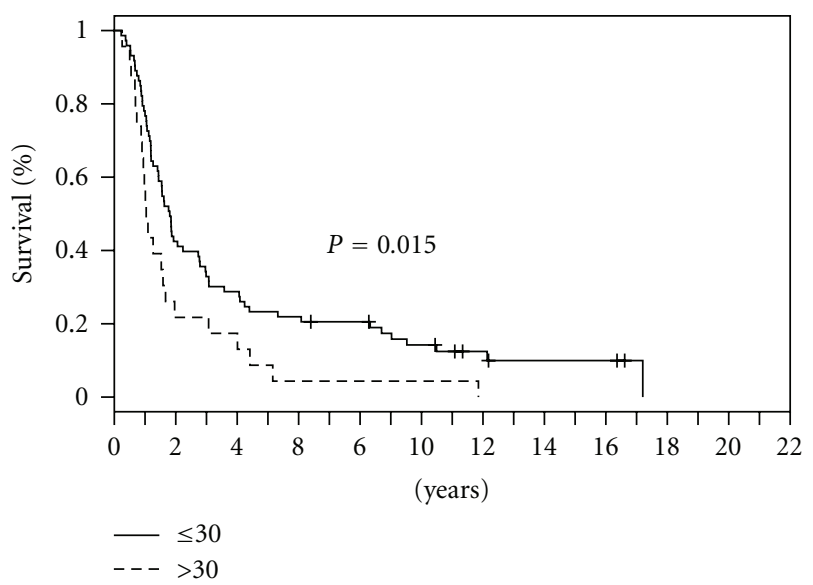

(d) PFS from metastasis

Figure 2: Overall survival (OS) from diagnosis (a) and from time of metastasis (b) and progression-free survival (PFS) from diagnosis (c) and from time to metastasis $(\mathrm{d})$ in patients with a BMI $\leq 30(n=73)$ or $>30(n=23)$. Survival was estimated using Kaplan-Meier method.

Second, and most surprisingly, in univariate and multivariate analyses, obesity was the only patient-related factor associated with significantly shorter OS and PFS from initial diagnosis and from when metastasis was diagnosed. Obesity also predicted shorter time from diagnosis to metastasis. Obesity has previously been reported as a risk factor for survival in patients diagnosed with earlier stages of breast cancer [30-32] and seems associated with higher stages at diagnosis [33], but, to the best of our knowledge, this is the first study reporting obesity as a risk factor for survival in patients with MBC. Other patient-related factors such as age, ethnicity, and menopausal status were not significant. Weights and obesity status were not available to us at the time of initial diagnosis, but recorded at the time of metastasis prior to first treatment. The median interval between initial diagnosis and time to metastasis was only approximately 3 years and can probably be considered a time frame where major weight changes are not expected to occur for the majority of patients [34]. We therefore felt comfortable to use the weight obtained at metastasis as the representative personal weight for each patient.

Third, among disease-related characteristics, stage at diagnosis was an independent predictor of time to metastasis. Histology and ER-status were not predictive in our cohort. Her-2/ neu was not part of the analysis since it was not routinely tested prior to 1999 . At the time of metastasis, in addition to stage at diagnosis, visceral metastasis also became an independent predictor of a higher hazard for progression and, suggestively, shortened OS. Higher stages at diagnosis are known to be associated with shorter time to recurrence and poorer survival $[35,36]$, and increased likelihood of occult metastasis in permissive niches such as the bone marrow and/or biologically more aggressive forms of cancer with increased burden of disease were suggested as plausible explanations for poorer survival at higher stages $[37,38]$. Also, visceral metastasis has been reported before as a predictor of poor survival in MBC [25-27]. The association of poorer outcomes with higher stages at initial diagnosis or 
visceral metastasis seems uniformly accepted, and our study supports these previous findings. The most important and novel finding was the negative effect of obesity on survival in $\mathrm{MBC}$, and its importance as independent predictor of time to metastasis and progression. To the best of our knowledge, these results provide first strong evidence that elevated BMI may contribute significantly to poor survival in $\mathrm{MBC}$ and these data were ascertained in the setting of HD-ASCT. While it has been reported that obesity is associated with higher stages of breast cancer at diagnosis [33], and that obesity negatively impacts various outcome factors in earlier stages of breast cancer [30-32], the effects of obesity have not been directly studied in MBC. Specifically in respect to locoregional breast cancer, two prospective studies identified obesity as an independent risk factor for overall mortality, distant and contralateral recurrence, and death from breast cancer [31, 32]. Similar findings for all presentations of breast cancer (some of which had MBC) were reported in the Prospective Analysis of Case-control studies on Environmental factors and Health (PACE) study [30].

In general, it is increasingly recognized that obesity and cancer are linked and that obesity not only confers a higher risk of cancer incidence but also increases cancer mortality as demonstrated in three large population-based cohort studies [39-41]. The reasons are poorly understood, but tumor growth stimulation by adipokines released from fatty tissue, inflammation [42-44], and especially in the case of breast cancer increased estrogen levels in obese postmenopausal females fueling tumor growth $[45,46]$ are implicated. Also, adjuvant undertreatment of obese patients, if chemotherapy is not strictly weight-based [4749], is increasingly recognized as important for early relapse. Against physicians' beliefs, chemotherapy in the obese is well tolerated, and, while dose increases proportional to weight do not confer a higher risk for toxicity [49, 50], underdosing in the obese breast cancer patient receiving adjuvant chemotherapy demonstrated increased risk for recurrence, shorter DFS or OS $[51,52]$. Despite these data, two recent surveys show that only $25 \%$ of breast cancer patients receive strictly weight-based dosing during adjuvant chemotherapy and that 19 of 44 cooperative group trials used dose limits for the obese $[47,48]$. In our study population, doses of previous adjuvant regimens were unknown, and suboptimal adjuvant treatment cannot be ruled out as a reason for faster time to metastasis in the obese. However, agents used for treatment in the metastatic setting and for HD-ASCT were dosed by actual body weight, and body surface areas were allowed to exceed $2 \mathrm{~m}^{2}$, such that underdosing does not appear to be the main reason for poorer outcome parameters. Further study limitations are absence of Her-2-neu as important prognostic marker, since it was not routinely available in the era of HD-ASCT, and patient population diversity. Selection bias may be present since not every patient deemed a candidate for HD-ASCT may have been referred or proceeded to HDASCT. Factors such as, but not limited to, physicians' or patients' treatment preferences, patients' performance status and socioeconomic status may have interfered.

Until now, obesity and its effect on outcomes in MBC have not been examined in depth, and this is the first report to implicate obesity in poorer outcomes. While this observation was made in the setting of HD-ASCT, conclusions about the contribution of HD-ASCT to longterm survivorship cannot be made, given retrospective study design, diverse study patient population, and mixed treatment algorithms. However, our results demonstrate that ultra-long-term survivorship can be achieved in the setting of more aggressive chemotherapy with HD-ASCT and that obesity was deleterious in MBC. In contrast to MBC, correction of unhealthy life-style behavior associated with obesity is the subject of intense exploration after adjuvant treatment for breast cancer, where patients are officially recognized as "breast cancer survivors." Results from the Women's Healthy Eating and Living (WHEL) study [53] suggest that obese patients can experience significant overall survival benefit if compliant with a combination of diet and exercise. We believe that our findings generate the hypothesis that obesity may be a potentially correctable patient-related adverse survival factor in $\mathrm{MBC}$ and, similar to approaches for locoregional stages of breast cancer, can serve as a target for intervention given the prospect of long-term survival in certain settings.

\section{References}

[1] P. Tai, E. Yu, V. Vinh-Hung, G. Cserni, and G. Vlastos, "Survival of patients with metastatic breast cancer: twentyyear data from two SEER registries," BMC Cancer, vol. 4, article 60, 2004.

[2] N. Wilcken and R. Dear, "Chemotherapy in metastatic breast cancer: a summary of all randomised trials reported 20002007," European Journal of Cancer, vol. 44, no. 15, pp. 22182225, 2008.

[3] C. E. Geyer, J. Forster, D. Lindquist et al., "Lapatinib plus capecitabine for HER2-positive advanced breast cancer," New England Journal of Medicine, vol. 355, no. 26, pp. 2733-2743, 2006.

[4] K. Miller, M. Wang, J. Gralow et al., "Paclitaxel plus bevacizumab versus paclitaxel alone for metastatic breast cancer," New England Journal of Medicine, vol. 357, no. 26, pp. 26662676, 2007.

[5] D. J. Slamon, B. Leyland-Jones, S. Shak et al., "Use of chemotherapy plus a monoclonal antibody against her2 for metastatic breast cancer that overexpresses HER2," New England Journal of Medicine, vol. 344, no. 11, pp. 783-792, 2001.

[6] R. Abraham, T. Nagy, P. E. Goss, and M. Crump, "High dose chemotherapy and autologous blood stem cell support in women with breast carcinoma and isolated supraclavicular lymph node metastases," Cancer, vol. 88, no. 4, pp. 790-795, 2000 .

[7] K. Antman, L. Ayash, A. Elias et al., "High-dose cyclophosphamide, thiotepa, and carboplatin with autologous marrow support in women with measurable advanced breast cancer responding to standard-dose therapy: analysis by age," Journal of the National Cancer Institute. Monographs, no. 16, pp. 91-94, 1994.

[8] L. J. Ayash, A. Elias, C. Wheeler et al., "Double dose-intensive chemotherapy with autologous marrow and peripheralblood progenitor-cell support for metastatic breast cancer: a feasibility study," Journal of Clinical Oncology, vol. 12, no. 1, pp. 37-44, 1994. 
[9] C. Gisselbrecht, J. M. Extra, J. P. Lotz et al., "Cyclophosphamide/mitoxantrone/melphalan (CMA) regimen prior to autologous bone marrow transplantation (ABMT) in metastatic breast cancer," Bone Marrow Transplantation, vol. 18, no. 5, pp. 857-863, 1996.

[10] N. Kröger, M. Frick, O. Gluz et al., "Randomized trial of single compared with tandem high-dose chemotherapy followed by autologous stem-cell transplantation in patients with chemotherapy-sensitive metastatic breast cancer," Journal of Clinical Oncology, vol. 24, no. 24, pp. 3919-3926, 2006.

[11] J. -P. Lotz, H. Curé, M. Janvier et al., "High-dose chemotherapy with haematopoietic stem cell transplantation for metastatic breast cancer patients: final results of the French multicentric randomised CMA/PEGASE 04 protocol," European Journal of Cancer, vol. 41, no. 1, pp. 71-80, 2005.

[12] P. Schmid, W. Schippinger, T. Nitsch, G. Huebner, R. Kreienberg, and W. Schultze, "Up-front tandem high-dose chemotherapy (HD) compared to standard chemotherapy with doxorubicin and paclitaxel (AT) in metastatic breast cancer (MBC): final results of a randomized trial," Journal of Clinical Oncology, vol. 22, p. 37S, 2004.

[13] J. J. Vredenburgh, B. Madan, D. Coniglio et al., "A randomized phase III comparative trial of immediate consolidation with high-dose chemotherapy and autologous peripheral blood progenitor cell support compared to observation with delayed consolidation in women with metastatic breast cancer and only bone metastases following intensive induction chemotherapy," Bone Marrow Transplantation, vol. 37, no. 11, pp. 1009-1015, 2006.

[14] J. J. Vredenburgh, D. Coniglio, G. Broadwater et al., "Consolidation with high-dose combination alkylating agents with bone marrow transplantation significantly improves disease-free survival in hormone-insensitive metastatic breast cancer in complete remission compared with intensive standard-dose chemotherapy alone," Biology of Blood and Marrow Transplantation, vol. 12, no. 2, pp. 195-203, 2006.

[15] M. Crump, S. Gluck, D. Tu et al., "Randomized trial of highdose chemotherapy with autologous peripheral-blood stemcell support compared with standard-dose chemotherapy in women with metastatic breast cancer: NCIC MA.16," Journal of Clinical Oncology, vol. 26, no. 1, pp. 37-43, 2008.

[16] E. A. Diaz-Canton, V. Valero, Z. Rahman et al., "Clinical course of breast cancer patients with metastases confined to the lungs treated with chemotherapy," Annals of Oncology, vol. 9, no. 4, pp. 413-418, 1998.

[17] O. Er, D. K. Frye, S. -W.C. Kau et al., "Clinical course of breast cancer patients with metastases limited to the liver treated with chemotherapy," Cancer Journal, vol. 14, no. 1, pp. 62-68, 2008.

[18] P. A. C. Greenberg, G. N. Hortobagyi, T. L. Smith, L. D. Ziegler, D. K. Frye, and A. U. Buzdar, "Long-term follow-up of patients with complete remission following combination chemotherapy for metastatic breast cancer," Journal of Clinical Oncology, vol. 14, no. 8, pp. 2197-2205, 1996.

[19] U. Güth, D. J. Huang, S. Dirnhofer, C. Rochlitz, and E. Wight, "Distant metastatic breast cancer as an incurable disease: a tenet with a need for revision," Cancer Journal, vol. 15, no. 1, pp. 81-86, 2009.

[20] G. N. Hortobagyi, "Can we cure limited metastatic breast cancer?" Journal of Clinical Oncology, vol. 20, no. 3, pp. 620-623, 2002.

[21] Jr. Sledge G.W., "Should we dream the impossible dream? The meaning of long-term survival in metastatic breast cancer," Journal of Clinical Oncology, vol. 14, no. 8, pp. 2191-2193, 1996.
[22] K. H. Antman, P. A. Rowlings, W. P. Vaughan et al., "Highdose chemotherapy with autologous hematopoietic stem-cell support for breast cancer in North America," Journal of Clinical Oncology, vol. 15, no. 5, pp. 1870-1879, 1997.

[23] A. D. Elias, J. Ibrahim, P. Richardson et al., "The impact of induction duration and the number of high-dose cycles on the long-term survival of women with metastatic breast cancer treated with high-dose chemotherapy with stem cell rescue: an analysis of sequential phase I/II trials from the Dana-Farber/Beth Israel STAMP program," Biology of Blood and Marrow Transplantation, vol. 8, no. 4, pp. 198-205, 2002.

[24] W. P. Peters, R. D. Dansey, J. L. Klein, and R. D. Baynes, "High-dose chemotherapy and peripheral blood progenitor cell transplantation in the treatment of breast cancer," Oncologist, vol. 5, no. 1, pp. 1-13, 2000.

[25] D. A. Rizzieri, J. J. Vredenburgh, R. Jones et al., "Prognostic and predictive factors for patients with metastatic breast cancer undergoing aggressive induction therapy followed by highdose chemotherapy with autologous stem-cell support," Journal of Clinical Oncology, vol. 17, no. 10, pp. 3064-3074, 1999.

[26] P. A. Rowlings, S. F. Williams, K. H. Antman et al., "Factors correlated with progression-free survival after high-dose chemotherapy and hematopoietic stem cell transplantation for metastatic breast cancer," Journal of the American Medical Association, vol. 282, no. 14, pp. 1335-1343, 1999.

[27] A. Schneeweiss, M. Hensel, P. Sinn et al., "Characteristics associated with long-term progression-free survival following high-dose chemotherapy in metastatic breast cancer and influence of chemotherapy dose," Annals of Oncology, vol. 13, no. 5, pp. 679-688, 2002.

[28] R. Brookmeyer and J. Crowley, "A confidence-interval for the median survival-time," Biometrics, vol. 38, pp. 29-41, 1982.

[29] S. E. Jones, "Metastatic breast cancer: the treatment challenge," Clinical Breast Cancer, vol. 8, no. 3, pp. 224-233, 2008.

[30] L. Dal Maso, A. Zucchetto, R. Talamini et al., "Effect of obesity and other lifestyle factors on mortality in women with breast cancer," International Journal of Cancer, vol. 123, no. 9, pp. 2188-2194, 2008.

[31] J. J. Dignam, K. Wieand, K. A. Johnson, B. Fisher, L. Xu, and E. P. Mamounas, "Obesity, tamoxifen use, and outcomes in women with estrogen receptor-positive early-stage breast cancer," Journal of the National Cancer Institute, vol. 95, no. 19, pp. 1467-1476, 2003.

[32] S. Loi, R. L. Milne, M. L. Friedlander et al., "Obesity and outcomes in premenopausal and postmenopausal breast cancer," Cancer Epidemiology Biomarkers and Prevention, vol. 14, no. 7, pp. 1686-1691, 2005.

[33] A. Macciò, C. Madeddu, G. Gramignano et al., "Correlation of body mass index and leptin with tumor size and stage of disease in hormone-dependent postmenopausal breast cancer: preliminary results and therapeutic implications," Journal of Molecular Medicine, vol. 88, pp. 677-686, 2010.

[34] J. M. Jakicic, B. H. Marcus, K. I. Gallagher, M. Napolitano, and W. Lang, "Effect of exercise duration and intensity on weight loss in overweight, sedentary women: a randomized trial," Journal of the American Medical Association, vol. 290, no. 10, pp. 1323-1330, 2003.

[35] A. Jemal, R. Siegel, E. Ward et al., "Cancer statistics, 2008," CA Cancer Journal for Clinicians, vol. 58, no. 2, pp. 71-96, 2008.

[36] W. A. Woodward, E. A. Strom, S. L. Tucker et al., "Changes in the 2003 American joint committee on cancer staging for breast cancer dramatically affect stage-specific survival," Journal of Clinical Oncology, vol. 21, no. 17, pp. 3244-3248, 2003. 
[37] S. Braun, K. Pantel, P. Müller et al., "Cytokeratin-positive cells in the bone marrow and survival of patients with stage I, II, or III breast cancer," New England Journal of Medicine, vol. 342, no. 8, pp. 525-533, 2000.

[38] S. A. Rabbani and A. P. Mazar, "Evaluating distant metastases in breast cancer: from biology to outcomes," Cancer and Metastasis Reviews, vol. 26, no. 3-4, pp. 663-674, 2007.

[39] E. E. Calle, C. Rodriguez, K. Walker-Thurmond, and M. J. Thun, "Overweight, obesity, and mortality from cancer in a prospectively studied cohort of U.S. Adults," New England Journal of Medicine, vol. 348, no. 17, pp. 1625-1638, 2003.

[40] G. K. Reeves, K. Pirie, V. Beral, J. Green, E. Spencer, and D. Bull, "Cancer incidence and mortality in relation to body mass index in the Million Women Study: cohort study," British Medical Journal, vol. 335, no. 7630, pp. 1134-1139, 2007.

[41] Y. -M. Song, J. Sung, and M. Ha, "Obesity and risk of cancer in postmenopausal Korean Women," Journal of Clinical Oncology, vol. 26, no. 20, pp. 3395-3402, 2008.

[42] D. R. Cottam, S. G. Mattar, E. Barinas-Mitchell et al., "The chronic inflammatory hypothesis for the morbidity associated with morbid obesity: implications and effect of weight loss," Obesity Surgery, vol. 14, no. 5, pp. 589-600, 2004.

[43] E. Fiorio, A. Mercanti, M. Terrasi et al., "Leptin/HER2 crosstalk in breast cancer: in vitro study and preliminary in vivo analysis," BMC Cancer, vol. 8, article 305, 2008.

[44] D. Sisci and E. Surmacz, "Crosstalk between IGF signaling and steroid hormone receptors in breast cancer," Current Pharmaceutical Design, vol. 13, no. 7, pp. 705-717, 2007.

[45] S. E. Hankinson, W. C. Willett, J. E. Manson et al., "Alcohol, height, and adiposity in relation to estrogen and prolactin levels in postmenopausal women," Journal of the National Cancer Institute, vol. 87, no. 17, pp. 1297-1302, 1995.

[46] S. Rinaldi, T. J. Key, P. H. M. Peeters et al., "Anthropometric measures, endogenous sex steroids and breast cancer risk in postmenopausal women: a study within the EPIC cohort," International Journal of Cancer, vol. 118, no. 11, pp. 2832$2839,2006$.

[47] C. G. Greenman, C. H. Jagielski, and J. J. Griggs, "Breast cancer adjuvant chemotherapy dosing in obese patients: dissemination of information from clinical trials to clinical practice," Cancer, vol. 112, no. 10, pp. 2159-2165, 2008.

[48] J. J. Griggs, E. Culakova, M. E. S. Sorbero et al., "Effect of patient socioeconomic status and body mass index on the quality of breast cancer adjuvant chemotherapy," Journal of Clinical Oncology, vol. 25, no. 3, pp. 277-284, 2007.

[49] P. Poikonen, C. Blomqvist, and H. Joensuu, "Effect of obesity on the leukocyte nadir in women treated with adjuvant cyclophosphamide, methotrexate, and fluorouracil dosed according to body surface area," Acta Oncologica, vol. 40, no. 1, pp. 67-71, 2001.

[50] P. Jenkins, S. Elyan, and S. Freeman, "Obesity is not associated with increased myelosuppression in patients receiving chemotherapy for breast cancer," European Journal of Cancer, vol. 43, no. 3, pp. 544-548, 2007.

[51] M. Colleoni, S. Li, R. D. Gelber et al., "Relation between chemotherapy dose, oestrogen receptor expression, and bodymass index," Lancet, vol. 366, no. 9491, pp. 1108-1110, 2005.

[52] G. L. Rosner, J. B. Hargis, D. R. Hollis et al., "Relationship between toxicity and obesity in women receiving adjuvant chemotherapy for breast cancer: results from Cancer and Leukemia Group B study 8541," Journal of Clinical Oncology, vol. 14, no. 11, pp. 3000-3008, 1996.
[53] J. P. Pierce, L. Natarajan, B. J. Cann, B. A. Parker, E. R. Greenberg, and S. W. Flatt, "The influence of a very high vegetable-fruit-fiber, low-fat diet on prognosis following treatment for breast cancer: results from the Women's Healthy Eating and Living (WHEL) randomized trial," Breast Cancer Research and Treatment, vol. 106, p. S16, 2007. 


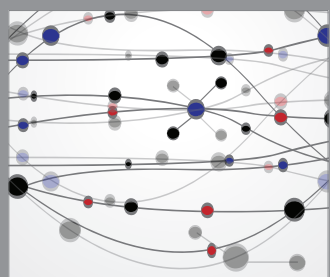

The Scientific World Journal
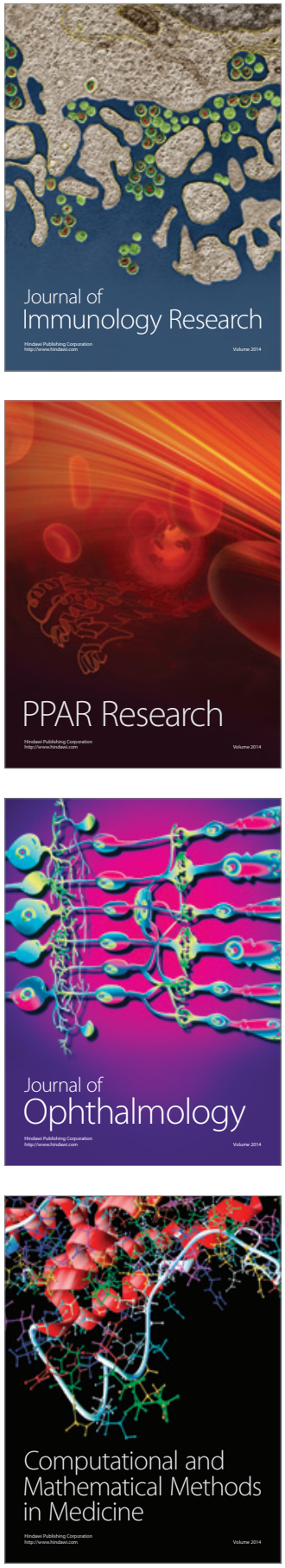

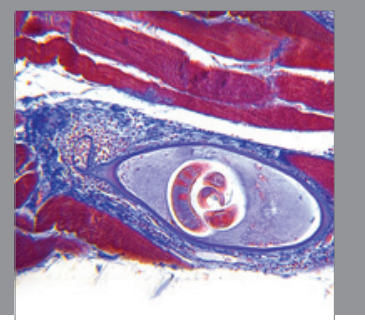

Gastroenterology

Research and Practice
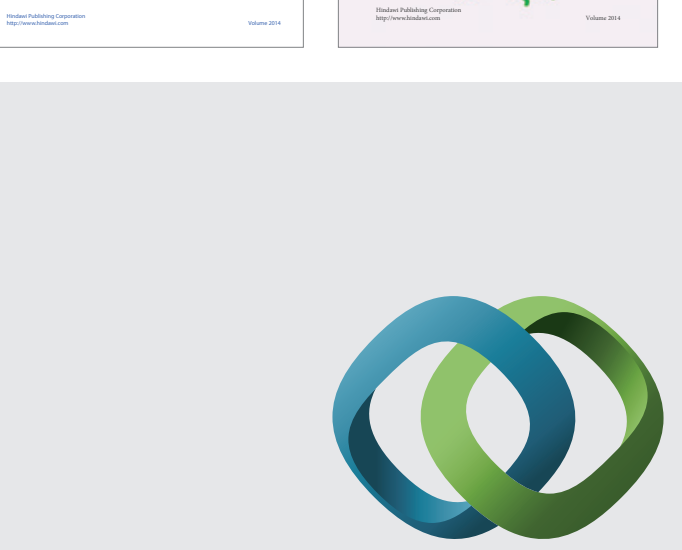

\section{Hindawi}

Submit your manuscripts at

http://www.hindawi.com
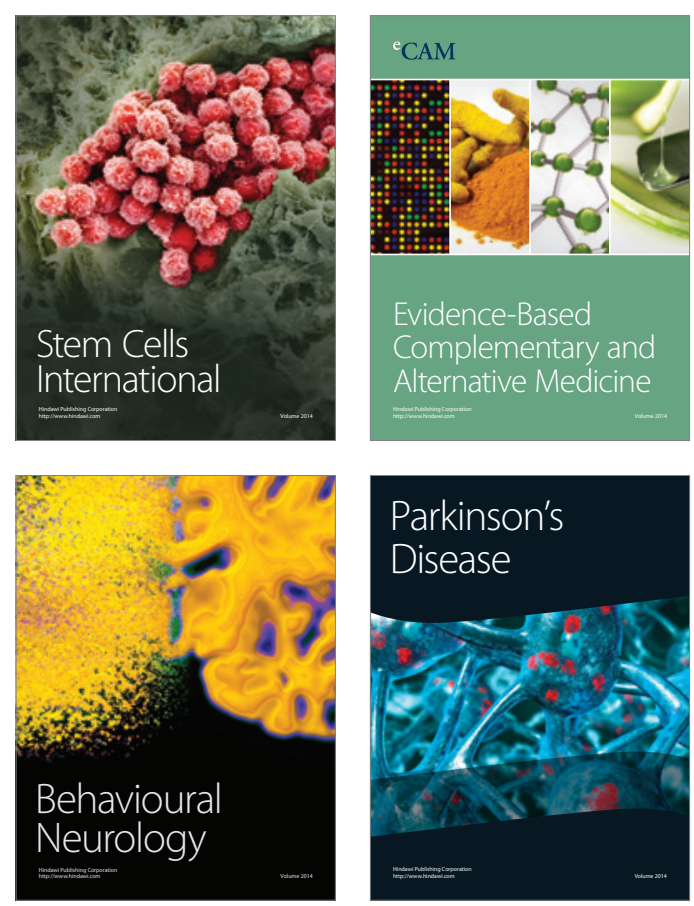

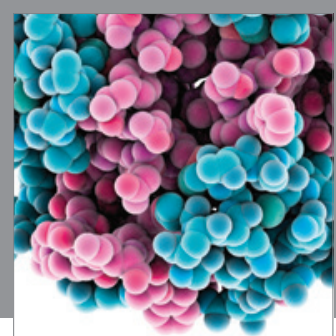

Journal of
Diabetes Research

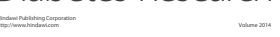

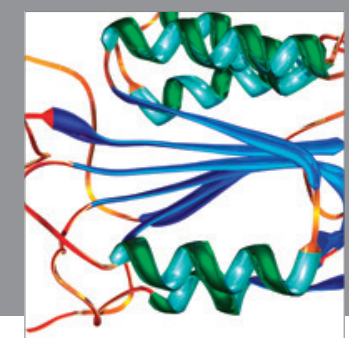

Disease Markers
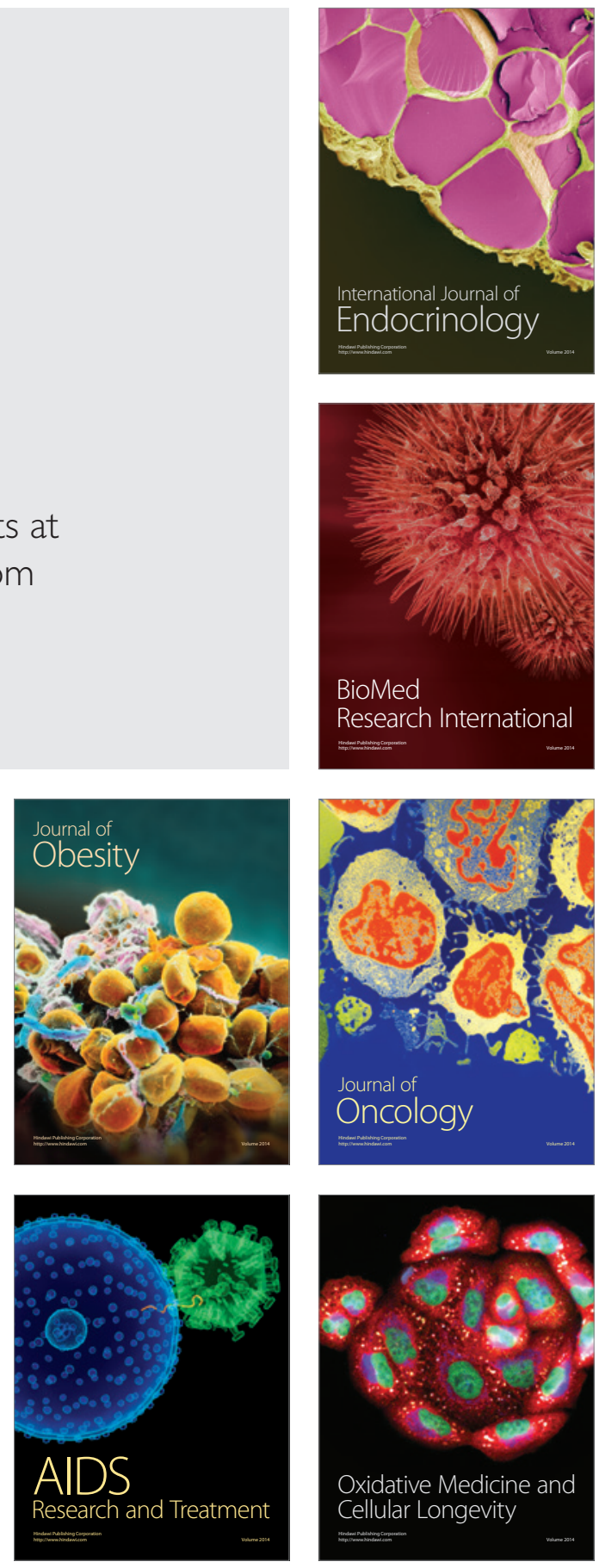